\title{
ON CERTAIN IMPROPER INTEGRALS
}

0. Summary. The functions $F$ and $G$ determined by integrals (1) and (2) are considered in the paper. Series expansions as well as asymptotic formulae for these functions are derived.

Integrals (14) and (15) are approximately calculated and expressed in terms of the functions $F$ and $G$. The error caused by the approximation is evaluated. The application of the integrals considered is illustrated by examples.

1. Fundamental equations. In the sequel, the integrals

$$
\begin{aligned}
& F(x, y)=\int_{0}^{\infty} \frac{e^{-u x} \cos u y}{u+a} d u, \\
& G(x, y)=\int_{0}^{\infty} \frac{e^{-u x} \sin u y}{u+a} d u
\end{aligned}
$$

will be considered with $a=\exp (i \pi / 4)$, real $y$ and $x>0$. Integrals (1) and (2) represent functions $F$ and $G$ of real variables $x$ and $y$.

The function $F$ is even, whereas the function $G$ is odd with respect to $y$, i. e. $F(x,-y)=F(x, y)$ and $G(x,-y)=-G(x, y)$.

It is easy to show that the functions $F$ and $G$ satisfy the Laplace equation

$$
\frac{\partial^{2} U}{\partial x^{2}}+\frac{\partial^{2} U}{\partial y^{2}}=0 .
$$

2. Series expansions. Substitution of

$$
\cos u y=\frac{1}{2}\left(e^{i u \nu}+e^{-i u \nu}\right)
$$

into (1) gives

$$
F(x, y)=\frac{1}{2}\left[\int_{0}^{\infty} \frac{e^{-u z} d u}{u+a}+\int_{0}^{\infty} \frac{e^{-u s} d u}{u+a}\right]
$$


where

$$
z=x+i y=r e^{i \theta}, \quad z^{*}=x-i y=r e^{i \theta}
$$

and

$$
r=\sqrt{x^{2}+y^{2}}, \quad \theta=\operatorname{arctg} \frac{y}{x}
$$

The integrals which appear in (3) can be expressed in terms of the exponential integral function, giving

$$
F(x, y)=\frac{1}{2}\left[-e^{a z} E i(-a z)-e^{a z^{*}} E i\left(-a z^{*}\right)\right]
$$

according to the formula No. 3.352 .4 in [1].

The exponential function as well as the exponential integral function which appear in (6) are complex. These functions can be represented in the form

$$
e^{a z}=A_{1}+i A_{2}, \quad-E i(-a z)=B_{1}+i B_{2}
$$

with $A_{1}, A_{2}, B_{1}, B_{2}$ determined later. Hence,

$$
-e^{a z} E i(-a z)=\left(A_{1}+i A_{2}\right)\left(B_{1}+i B_{2}\right) \text {. }
$$

Inserting $z=r e^{i \theta}$ into the expression

$$
e^{a s}=\sum_{k=0}^{\infty} \frac{a^{k} z^{k}}{k !}
$$

we obtain

$$
A_{1}=\sum_{k=0}^{\infty} \frac{a^{k} r^{k} \cos k \theta}{k !}, \quad A_{2}=\sum_{k=1}^{\infty} \frac{a^{k} r^{k} \sin k \theta}{k !}
$$

if Euler's identity $e^{i k \theta}=\cos k \theta+i \sin k \theta$ is applied. Using $a^{k+4}=-a^{k}$, equations (8) become

$$
\begin{aligned}
& A_{1}=1+a c_{1}+a^{2} c_{2}+a^{3} c_{3}+a^{4} c_{4}, \\
& A_{2}=a s_{1}+a^{2} s_{2}+a^{3} s_{3}+a^{4} s_{4},
\end{aligned}
$$

where

$$
\begin{aligned}
& c_{m}=\sum_{k=0}^{\infty}(-1)^{k} \frac{r^{m+4 k} \cos (m+4 k) \theta}{(m+4 k) !} \\
& 8_{m}=\sum_{k=0}^{\infty}(-1)^{k} \frac{r^{m+4 k} \sin (m+4 k) \theta}{(m+4 k) !}
\end{aligned}
$$


for $m=1,2,3,4$. Substitution of

$$
\begin{array}{cc}
a=\frac{1}{\sqrt{2}}(1+i), & a^{2}=i \\
a^{3}=\frac{1}{\sqrt{2}}(-1+i), & a^{4}=-1
\end{array}
$$

into (9) gives

$$
\begin{aligned}
& A_{1}=\left[1-c_{4}+\frac{1}{\sqrt{2}}\left(c_{1}-c_{3}\right)\right]+i\left[c_{2}+\frac{1}{\sqrt{2}}\left(c_{1}+c_{3}\right)\right], \\
& A_{2}=\left[-s_{4}+\frac{1}{\sqrt{2}}\left(s_{1}-s_{3}\right)\right]+i\left[s_{2}+\frac{1}{\sqrt{2}}\left(s_{1}+s_{3}\right)\right]
\end{aligned}
$$

The functions $B_{1}, B_{2}$ associated with $-E i(-a z)$ are derived in a similar way if the series [5]

$$
E i(z)=C+\ln (-z)+\sum_{k=1}^{\infty} \frac{z^{k}}{k \cdot k !}
$$

is used, where $C=0,5772 \ldots$ is Euler's constant. We obtain

$$
\begin{aligned}
& B_{1}=\left[-C-\ln r+c_{4}^{\prime}+\frac{1}{\sqrt{2}}\left(c_{1}^{\prime}-c_{3}^{\prime}\right)\right]+i\left[-\frac{\pi}{4}-c_{2}^{\prime}+\frac{1}{\sqrt{2}}\left(c_{1}^{\prime}+c_{3}^{\prime}\right)\right], \\
& B_{2}=\left[-\theta+s_{4}^{\prime}+\frac{1}{\sqrt{2}}\left(s_{1}^{\prime}-s_{3}^{\prime}\right)\right]+i\left[-s_{2}^{\prime}+\frac{1}{\sqrt{2}}\left(s_{1}^{\prime}+s_{3}^{\prime}\right)\right],
\end{aligned}
$$

where

$$
\begin{aligned}
& c_{m}^{\prime}=\sum_{k=0}^{\infty}(-1)^{k} \frac{r^{m+4 k} \cos (m+4 k) \theta}{(m+4 k)(m+4 k) !} \\
& s_{m}^{\prime}=\sum_{k=0}^{\infty}(-1)^{k} \frac{r^{m+4 k} \sin (m+4 k) \theta}{(m+4 k)(m+4 k) !}
\end{aligned}
$$

for $m=1,2,3,4$.

The relationships for $e^{a z^{*}}$ and $-E i\left(-a z^{*}\right)$ are derived if $\theta$ is replaced by $-\theta$, giving

$$
-e^{a z^{*}} E i\left(-a z^{*}\right)=\left(A_{1}-i A_{2}\right)\left(B_{1}-i B_{2}\right),
$$

since $A_{1}$ and $B_{1}$ are even, whereas $A_{2}$ and $B_{2}$ are odd with respect to $\theta$. Inserting: (7) and (10) into (6), we obtain

$$
F(x, y)=A_{1} B_{1}-A_{2} B_{2} \text {. }
$$


Now we consider the function $G$. Substitution of

$$
\sin u y=\frac{1}{2 i}\left(e^{i u y}-e^{-i u y}\right)
$$

into (2) gives

$$
G(x, y)=\frac{1}{2 i}\left[e^{a z} E i(-a z)-e^{a *} E i\left(-a z^{*}\right)\right] .
$$

Inserting (7) and (10) into (11), we have

$$
G(x, y)=-\left(A_{1} B_{2}+A_{2} B_{1}\right) .
$$

For small $r$ we obtain

$$
\begin{aligned}
& F(x, y)=-0,577+\ln \frac{1}{r}+O(r), \\
& G(x, y)=\theta+O(r) .
\end{aligned}
$$

From the relationships derived the numerical values of the functions $F$ and $G$ can be computed using digital computers. The tables of these functions are given in [2] and [3].

3. Asymptotic formulae. The asymptotic formulae of the functions $F$ and $G$ are obtained from the asymptotic formula for the integral exponential function [5]:

$$
E i(z) \sim \frac{e^{z}}{z} \sum_{k=0}^{\infty} \frac{k !}{z^{k}}
$$

We have

$$
-e^{a \varepsilon} E i(-a z) \sim \sum_{k=0}^{\infty}(-1)^{k} \frac{k !}{(a z)^{k+1}}
$$

whence

$$
-e^{a z} E i(-a z) \sim \sum_{k=0}^{\infty}(-1)^{k} \frac{k ! \exp [-i(k+1) \theta]}{(a r)^{k+1}}
$$

and

$$
-e^{a z^{*}} E i\left(-a z^{*}\right) \sim \sum_{k=0}^{\infty}(-1)^{k} \frac{k ! \exp [i(k+1) \theta]}{(a r)^{k+1}}
$$

where $r$ and $\theta$ are taken from (5). 
Substitution of (12) and (13) into (6) and (11) gives

$$
\begin{aligned}
& F(x, y) \sim {\left[\frac{\sqrt{2} \cos \theta}{2 r}-\frac{\sqrt{2} \cos 3 \theta}{r^{3}}+\frac{6 \cos 4 \theta}{r^{4}}-\frac{12 \sqrt{2} \cos 5 \theta}{r^{5}}+\ldots\right]+} \\
&+i\left[-\frac{\sqrt{2} \cos \theta}{2 r}+\frac{\cos 2 \theta}{r^{2}}-\frac{\sqrt{2} \cos 3 \theta}{r^{3}}+\frac{12 \sqrt{2} \cos 5 \theta}{r^{5}}+\ldots\right], \\
& G(x, y) \sim {\left[\frac{\sqrt{2} \sin \theta}{2 r}-\frac{\sqrt{2} \sin 3 \theta}{r^{3}}+\frac{6 \sin 4 \theta}{r^{4}}-\frac{12 \sqrt{2} \sin 5 \theta}{r^{5}}+\ldots\right]+} \\
&+i\left[-\frac{\sqrt{2} \sin \theta}{2 r}+\frac{\sin 2 \theta}{r^{2}}-\frac{\sqrt{2} \sin 3 \theta}{r^{3}}+\frac{12 \sqrt{2} \sin 5 \theta}{r^{5}}+\ldots\right] .
\end{aligned}
$$

4. Approximate evaluation of two integrals. Now we consider the integrals

$$
\begin{aligned}
& I_{1}=\int_{0}^{\infty} \frac{e^{-u x} \cos u y}{\mu u+\sqrt{u^{2}+i}} d u, \\
& I_{2}=\int_{0}^{\infty} \frac{e^{-u x} \sin u y}{\mu u+\sqrt{u^{2}+i}} d u,
\end{aligned}
$$

where $y$ is real, $x>0$ and $\mu>0$. It is possible to express these integrals in terms of the function $F$ or $G$ if $x \geqslant 20$ and $\mu \geqslant 50$.

In order to evaluate the last integrals, we neglect the term $u^{2}$ involved in the root. Then the error resulting becomes

$$
\varepsilon=\left|\int_{0}^{\infty} \frac{e^{-u x} \cos u y}{\mu u+a} d u-I_{1}\right|,
$$

where $a=\exp (i \pi / 4)$. Hence,

$$
\varepsilon \leqslant \int_{0}^{\infty} \frac{u^{2} e^{-u x}}{\left|(\mu u+a)\left(\mu u+\sqrt{u^{2}+i}\right)\left(\sqrt{u^{2}+i}+a\right)\right|} d u .
$$

From

$$
|\mu u+a|=\left|\mu u+\frac{1}{\sqrt{2}}+i \frac{1}{\sqrt{2}}\right|=\sqrt{(\mu u)^{2}+\sqrt{2} \mu u+1}
$$

we obtain

$$
|\mu u+a| \geqslant \sqrt{(\mu u)^{2}+1}
$$


if $u \geqslant 0$. Similarly, from

$$
\sqrt{u^{2}+i}=\sqrt{\frac{\sqrt{u^{4}+1}+u^{2}}{2}}+i \sqrt{\frac{\sqrt{u^{4}+1}-u^{2}}{2}}
$$

we obtain

$$
\left|\mu u+\sqrt{u^{2}+i}\right| \geqslant \sqrt{(\mu u)^{2}+1}
$$

if $u \geqslant 0$ and $\left|\sqrt{u^{2}+i}+a\right| \geqslant 1$ for each $u$. Therefore the expression (16) becomes

$$
\varepsilon \leqslant \int_{0}^{\infty} \frac{u^{2} e^{-u x}}{\mu^{2} u^{2}+1} d u \leqslant \frac{1}{\mu^{2}} \int_{0}^{\infty} e^{-u x} d u,
$$

whence

$$
\varepsilon \leqslant \frac{1}{\mu^{2} x} .
$$

For $\mu \geqslant 50$ and $x \geqslant 20$ we obtain $\varepsilon \leqslant 2 \cdot 10^{-5}$.

Thus we have

$$
I_{1}=\int_{0}^{\infty} \frac{e^{-u x} \cos u y}{\mu u+a} d u,
$$

approximately. It is easy to show that the result derived is also valid for the integral $I_{2}$. Therefore we obtain

$$
I_{2}=\int_{0}^{\infty} \frac{e^{-u x} \sin u y}{\mu u+a} d u,
$$

approximately, with the same error. The error resulting in the both cases is less than $2 \cdot 10^{-5}$.

Introducing a new variable $v=\mu u$ into (17) and (18), we derive the approximate relationships

$$
\begin{aligned}
& \int_{0}^{\infty} \frac{e^{-u x} \cos u y}{\mu u+\sqrt{u^{2}+i}} d u=\frac{1}{\mu} F^{\prime}\left(\frac{x}{\mu}, \frac{y}{\mu}\right), \\
& \int_{0}^{\infty} \frac{e^{-u x} \sin u y}{\mu u+\sqrt{u^{2}+i}} d u=\frac{1}{\mu} G\left(\frac{x}{\mu}, \frac{y}{\mu}\right) .
\end{aligned}
$$

5. Applications. The integrals considered occur in problems concerning the electromagnetic field in a semi-infinite ferromagnetic body due to alternating currents in parallel conductors which are placed in a non- 
-conducting medium above the boundary surface of the body, if its permeability is assumed to be constant ([2], [3], [4], [6]). The examples which ilustrate the application of the integrals are:

1. The unit-length external impedance of the conductor $l$ placed above the boundary surface of a ferromagnetic body is given by

$$
Z_{e l}=\frac{i \omega \mu_{0}}{\pi}\left[\mu_{r} \int_{0}^{\infty} \frac{e^{-2 h} k k u d u}{\mu_{r} u+\sqrt{u^{2}+i}}+\frac{1}{2} \ln \frac{2 h_{l}}{r_{l}}\right]
$$

whereas the unit-length mutual impedance of the conductors $l$ and $m$ is

$$
Z_{l m}=\frac{i \omega \mu_{0}}{\pi}\left[\mu_{r} \int_{0}^{\infty} \frac{e^{-\left(h_{l}+h_{m}\right) k u} \cos k a_{l m}}{\mu_{r} u+\sqrt{u^{2}+i}} d u+\frac{1}{2} \ln \sqrt{\frac{\left(h_{l}+h_{m}\right)^{2}+a_{l m}^{2}}{\left(h_{l}-h_{m}\right)^{2}+a_{l m}^{2}}}\right],
$$

in accordance with [2]. The notation accepted in the last equations is: $\omega$ - angular frequency, $\mu_{r}$ - relative permeability of ferromagnetic medium, $h$ - distance from conductor $l$ to boundary surface, $r_{l}$ - radius of conductor $l, a_{l m}$ - horizontal distance between conductors $l$ and $m$, $k=\sqrt{\omega \mu_{0} \mu_{r} \gamma}, \mu_{0}$ - permeability of vacuum, $\gamma$ - conductivity of ferromagnetic medium.

The external and mutual impedances become

$$
\begin{gathered}
Z_{e l}=\frac{i \omega \mu_{0}}{\pi}\left[F\left(\frac{2 k}{\mu_{r}} h_{l}, 0\right)+\frac{1}{2} \ln \frac{2 h_{l}}{r_{l}}\right], \\
Z_{l m}=\frac{i \omega \mu_{0}}{\pi}\left\{F\left[\frac{k}{\mu_{r}}\left(h_{l}+h_{m}\right), \frac{k}{\mu_{r}} a_{l m}\right]+\frac{1}{2} \ln \sqrt{\frac{\left(h_{l}+h_{m}\right)^{2}+a_{l m}^{2}}{\left(h_{l}-h_{m}\right)^{2}+a_{l m}^{2}}}\right\},
\end{gathered}
$$

approximately, if (19) is used. The external and mutual impedances occur in relationships for eddy-current loss in the ferromagnetic body [2]. The last equations are valid for $\mu_{r} \geqslant 50$ and $2 h k \geqslant 20$, where $h$ is the minimum value of $h_{l}$ and $h_{m}$.

2. The electric intensity at the boundary surface of the ferromagnetic body is [2]

$$
E=-\frac{i \omega \mu_{0} \mu_{r} I}{\pi} \int_{0}^{\infty} \frac{e^{-h_{l} k u} \cos k y u}{\mu_{r} u+\sqrt{u^{2}+i}} d u,
$$

where $I$ is the complex current in the conductor $l$ and $y$ is the horizontal distance from the conductor $l$ to the point under consideration. Using (19), the electric intensity becomes

approximately.

$$
E=-\frac{i \omega \mu_{0} I}{\pi} F\left(\frac{k}{\mu_{r}} h_{l}, \frac{k}{\mu_{r}} y\right)
$$


An approximate relationship for the tangential component of the magnetic intensity at the boundary surface takes the form (cf. [2])

$$
H=-\frac{k I e^{i \pi / 4}}{\pi \mu_{r}} F\left(\frac{k}{\mu_{r}} h_{l}, \frac{k}{\mu_{r}} y\right) .
$$

From the last expressions, the density of complex power on the boundary surface can be determined; as result we obtain

$$
\Pi=\frac{\omega \mu_{0} k|I|^{2} e^{i \pi / 4}}{\pi^{2} \mu_{r}}\left|F\left(\frac{k}{\mu_{r}} h_{l}, \frac{k}{\mu_{r}} y\right)\right|^{2} 。
$$

3. The function $G$ appears in relationships for the electric and magnetic intensities when the current flows along a ribbon conductor which is placed in the non-conducting medium parallel to the boundary surface (cf. [3]).

\section{References}

[1] И. С. Градштейн п И. М. Рыжик, Таблицы интегралов, сумм, рядов и произведений, изд. 4, Москва 1963.

[2] M. Krakowski, Active power loss in the ferromagnetic medium due to currents in a multi-conductor system, Archiwum Elektrotechniki 19 (1970), p. 85-105.

[3] M. Krakowski, Distribution of active power on surface of ferromagnetic medium due to currents in parallel conductors, Archiwum Elektrotechniki 19 (1970), p. 525 -538.

[4] А. Н. Кравченко и Л. П. Нужник, Электромагнитное поле и потери системы прямолинейных бесконечно длинных проводов с переменным током, Киев 1967, р. 61-72.

[5] N. N. Lebiediew, Funkcje specjalne i ich zastosowania, PWN, Warszawa 1957.

[6] H. Poritsky and R. P. Jerrard, Eddy current losses in a semi-infinite solid due to a nearby alternating current, Trans. AIEE 73 (1954), Part I, p. 97-106.

Received on 14. 8. 1970

M. KRAKOWSKI (LODZ)

\section{O PE WNYCH CAEKACH NIE WLASCI WYCH}

\section{STRESZCZENIH}

W pracy rozpatrzono funkoje $F$ i $G$ określone przy pomocy całek (1) i (2) oraz wyznaczono szeregi $i$ wzory asymptotyczne dla tych funkcji.

Obliczono w sposób przybliżony całki (14) i (15), wyrażając je w zaleźności od funkcji $F$ i $G$. Oszacowano błąd popełniony w wyniku przyjetych przybliżeń. Zastosowanie rozpatrywanych całek zilustrowano przykładami. 